\title{
NORMAL POINTS FOR GENERIC HYPERBOLIC MAPS
}

\author{
MARK POLlicotT \\ Warwick University \\ To Michael Misiurewicz on the occasion of his 60th birthday
}

\section{INTRODUCTION}

Given a continuous transformation on a compact space we call a point normal if the ergodic averages of any continuous functions converge. For example, the familiar notion of a normal number $0<\xi<1$ in the context of number theory is one which is normal in the above sense for all of the transformations $T:[0,1) \rightarrow[0,1)$ given by $T(\xi)=d \xi(\bmod 1)$ for each $d \geq 2$.

In this note, we want to consider the question of when a specific point is normal for typical transformations, in some suitable sense. We begin by considering a particularly simple setting. Let $f_{(\lambda)}: K \rightarrow K$, for $\lambda \in(-\epsilon, \epsilon)$, be a family of $C^{1}$ orientation preserving expanding maps of the unit circle $K=\mathbb{R} / \mathbb{Z}$ of degree $d \geq 2$ which are perturbations of the standard linear map $f_{(0)}: K \rightarrow K$ given by $f_{(0)}(\xi)=d \xi(\bmod 1)$.

A simple result is the following.

Theorem. Fix a point $x \in M$. For typical non-trivial families $f_{(\lambda)}: K \rightarrow K$ we have that there exists $\epsilon=\epsilon(x)>0$ such that for almost all $\lambda \in(-\epsilon, \epsilon)$, with respect to Lebesgue measure, we have that the $f_{(\lambda)}$ orbit of $x$ is normal, i.e., there exists a measure $m_{(\lambda)}$ on $K$ such that

$$
\lim _{n \rightarrow+\infty} \frac{1}{n} \sum_{j=1}^{n} g\left(f_{(\lambda)}^{j} x\right)=\int g(\xi) d m_{(\lambda)}(\xi),
$$

for all continuous functions $g: K \rightarrow \mathbb{R}$.

The somewhat vague "typicality" hypothesis of the theorem can be more precisely formulated as follows. It is well known that there is a natural family of conjugating maps $\pi_{(\lambda)}: K \rightarrow K$ such that $f_{(\lambda)} \pi_{(\lambda)}=\pi_{(\lambda)} f_{(0)}$ (and $\pi_{(0)}$ is the identity). We require that $x$ satisfies $\frac{d \pi_{(\lambda)}(x)}{d \lambda} \neq 0$.

Furthermore, the measure $m_{(\lambda)}$ describing distribution the of the orbit is simply the unique measure of maximal entropy for $f_{(\lambda)}$. In particular, in the special case of the linear map $f_{(0)}$ the measure of maximal entropy $m_{(0)}$ is precisely the Haar measure on $K$.

The above theorem is probably best understood by considering a specific example, as in the next section. In later sections we consider the generalization to diffeomorphisms and flows.

Typeset by $\mathcal{A} \mathcal{M S}-\mathrm{TEX}_{\mathrm{E}} \mathrm{X}$ 
In a recent paper [4] of Faller and Pfisier the authors studied a certain parameterised family of piecewise linear maps of the interval for which the measure of maximal entropy was absolutely continuous. They considered a reference point and showed that for almost all values in the parameter space (with respect to Lebesgue measure) the orbits are normal. In an earlier paper [2], Bruin showed that for a certain paramterized family of tent maps one has that almost all parameter values the critical point $c$ has an orbit which is normal. Finally, part of the motivation for Bruin's result was a paper of Brucks and Misiurewicz [1] showing that the orbit of orbit of the critical point is dense for almost all parameter values.

\section{EXAMPLE}

Consider a family of expanding maps $f_{(\lambda)}: K \rightarrow K$ on the unit circle defined by $f_{(\lambda)}(\xi)=2 \xi+p(\lambda, \xi)(\bmod 1)$ where:

(1) $p(\lambda, \xi)$ is bianalytic on $(-\delta, \delta) \times K$; and

(2) $p(0, \xi)=0$ for each $\xi \in K$.

In particular, $f_{(0)}: K \rightarrow K$ is the usual doubling map $f_{(0)}(\xi)=2 \xi(\bmod 1)$. If we assume that $\partial p(\lambda, \xi) / \partial \xi>-1$, for $|\lambda|<\epsilon$ and $\xi \in K$, then $f_{(\lambda)}$ is expanding. We denote by $\pi_{(\lambda)}: K \rightarrow K$ the natural topological conjugacy between $f_{(\lambda)}$ and $f_{(0)}$, i.e., $\pi_{(\lambda)}$ is a homeomorphism such that

$$
\pi_{(\lambda)}\left(f_{(0)} \xi\right)=f_{(\lambda)}\left(\pi_{(\lambda)} \xi\right) \text { for each } \xi \in K
$$

The map $\pi_{(0)}$ will be the identity. However, if $\lambda \neq 0$ then the map $\pi_{(\lambda)}: K \rightarrow K$ will be Hölder continuous, but not usually $C^{1}$. However, there is a $C^{1}$ dependence $(-\epsilon, \epsilon) \ni \lambda \mapsto \pi_{(\lambda)} \in C^{0}(K, K)$, as we shall recall in $\S 2$. In particular, for a fixed reference point $x \in K$ the map $(-\epsilon, \epsilon) \ni \lambda \mapsto \pi_{(\lambda)}(x)$ is $C^{1}$.

To be even more concrete, we could choose $p(\lambda, \xi)=\lambda \sin (2 \pi \xi)$.
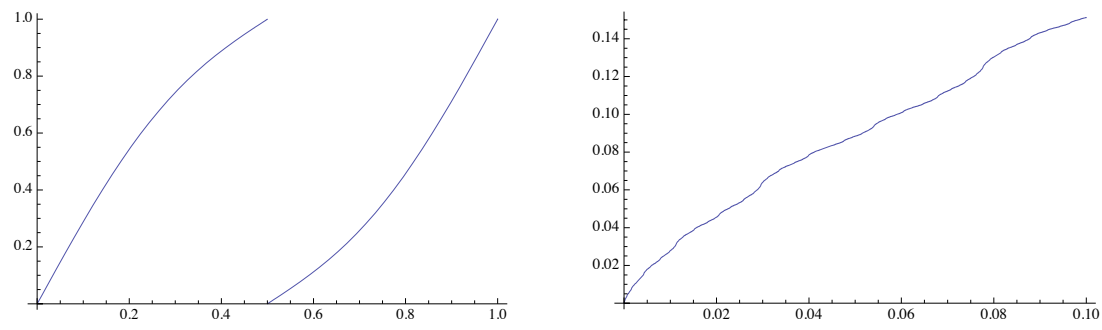

Figure 1:. (a) The graph of $f_{\left(\frac{1}{10}\right)}(x)=2 x+\frac{1}{10} \sin (2 \pi x)(\bmod 1)$; and (b) the graph of the (non-differentiable) conjugating map $\pi_{\left(\frac{1}{10}\right)}$.

In this case, the point $x=0$, for example, doesn't move under the perturbation and is thus exceptional. To study the behaviour of the other points, we can differentiate (1.1) with respect to $\lambda$ to write

$$
\left.\frac{\partial \pi_{(\lambda)}(2 x)}{\partial \lambda}\right|_{\lambda=0}=\left.\underbrace{\left.\frac{\partial f_{(0)}(\xi)}{\partial \xi}\right|_{\xi=x}}_{=2} \frac{\partial \pi_{(\lambda)}(x)}{\partial \lambda}\right|_{\lambda=0}+\underbrace{\frac{f_{(\lambda)}(x)}{\partial \lambda}}_{=\sin (2 \pi 2 \xi)}
$$


We can then iterate the identity (1.2) to deduce that the solution has series expansion

$$
\begin{aligned}
\left.\frac{\partial \pi_{(\lambda)}(x)}{\partial \lambda}\right|_{\lambda=0} & =\lim _{N \rightarrow+\infty}\left(-\sum_{n=1}^{N} 2^{-n} \sin \left(2 \pi 2^{n} x\right)+\left.2^{-N} \frac{d \pi_{(\lambda)}\left(2^{N} x\right)}{d \lambda}\right|_{\lambda=0}\right) \\
& =-\sum_{n=1}^{\infty} 2^{-n} \sin \left(2 \pi 2^{n} x\right)
\end{aligned}
$$

which is $\alpha$-Hölder continuous as a function of $x$, for any $0<\alpha<1$, but not $C^{1}$.
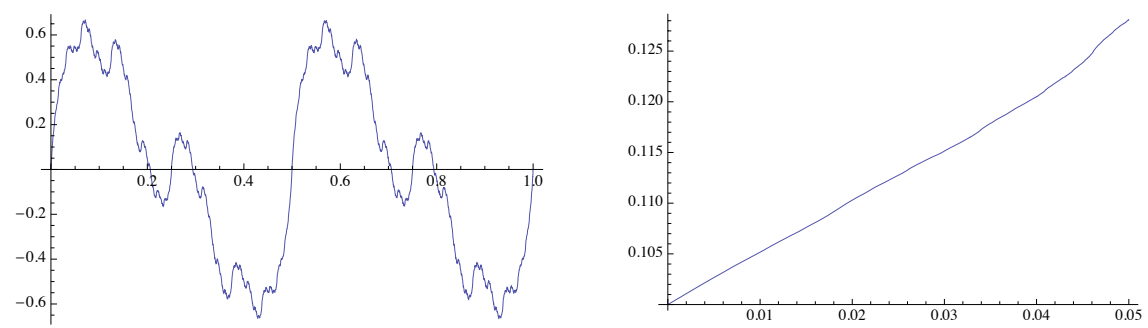

Figure 2. (a) The graph of the non-differentiable function $\left.x \mapsto \frac{\partial \pi_{(\lambda)}(x)}{\partial \lambda}\right|_{\lambda=0}$; and (b) the graph of the map $\lambda \mapsto \pi_{(\lambda)}\left(\frac{1}{10}\right)$ (i.e., with $\left.x=\frac{1}{10}\right)$, which is analytic for $|\lambda|$ sufficiently small.

With the exception of the points for which the above derivative is zero, we can choose $\epsilon(x)>0$ for which the derivative $\left.\frac{\partial \pi_{(\lambda)}(x)}{\partial \lambda}\right|_{\lambda=0}$ remains non-zero for $\lambda \in(-\epsilon(x), \epsilon(x))$.

\section{Conjugating MAP FOR EXPANDing MAPS}

We say that the map $f_{(\lambda)}: K \rightarrow K, \lambda \in(-\epsilon, \epsilon)$, is expanding if $\inf _{\xi}\left|f_{(\lambda)}^{\prime}(\xi)\right|>1$.

Let $C^{0}(K, K)$ be the space of continuous functions from $K$ to iteself. Given $0<\delta<\frac{1}{2}$, we can consider a neighbourhood $\mathcal{U} \subset C^{0}(K, K)$ of the identity map on $K$ which is naturally identified with $C^{0}(K,(-\delta, \delta))$ by $\pi(x)=x+h(x)$, where $h \in C^{0}(K,(-\delta, \delta))$, say, for $\delta>0$ sufficiently small. Using the natural inclusion $C^{0}(K,(-\delta, \delta)) \subset C^{0}(K, \mathbb{R})$ we can interpret $\mathcal{U}$ in terms of Banach manifolds. In particuar, writing

$$
(-\epsilon, \epsilon) \ni \lambda \mapsto \pi_{(\lambda)}(x)=: x+h_{(\lambda)}(x)
$$

we say that $(-\epsilon, \epsilon) \ni \lambda \mapsto \pi_{(\lambda)}$ is $C^{1}$ if for any bounded linear map $L \in C^{0}(K, \mathbb{R})^{*}$ we have that $(-\epsilon, \epsilon) \ni \lambda \mapsto L\left(h_{(\lambda)}\right) \in \mathbb{R}$ is $C^{1}$.

The next result is fairly standard.

\section{Proposition 2.1 (Structural Stability).}

(1) There exist conjugating homeomorphisms $\pi_{(\lambda)} \in C^{0}(K, K)$ such that

$$
\pi_{(\lambda)} \circ f_{(\lambda)}=f_{(0)} \circ \pi_{(\lambda)}, \text { for each } \lambda \in(-\epsilon, \epsilon) ;
$$

(2) $m_{(\lambda)}=\pi_{(\lambda)_{*}} m_{(0)}$ (i.e., for any continuous function $F: K \rightarrow \mathbb{R}$ we have that $\left.\int F \circ \pi_{(\lambda)}^{-1} d m_{(\lambda)}=\int F d m_{(0)}\right)$;

(3) The map $(-\epsilon, \epsilon) \ni t \mapsto \pi_{(\lambda)} \in C^{0}(K, K)$ is $C^{1}$. 
Proof. There is a very accessible account of the construction of structural stability and part (1) for interval maps in [6] (using the fixed point method). This can be adapted to show the first part of the proof. We can assume without loss of generality that $f_{(0))}$ fixes $0 \in \mathbb{R} / \mathbb{Z}$ and then we can consider $C^{0}(I, I)$ in place of $C^{0}(K, K)$, where $I=[0,1]$. Let $a_{m}(1 \leq m \leq k)$ be the preimages. One initially looks for fixed points $\pi \in C^{0}(I, I)$ to the maps $f_{(\lambda)}^{*}(\cdot): C^{0}(I, I) \rightarrow C^{0}(I, I)$ defined by

$$
\left.f_{(\lambda)}^{*}(\pi)(x)=\frac{1}{k} \pi\left(f_{(\lambda)} x\right)\right)+\frac{m}{k} \text { for } a_{m} \leq x \leq a_{m+1} .
$$

The existence of the fixed point follows using the contraction mapping theorem, cf. $[6]$.

To prove part (2), recall that since the topological entropy is preserved by topological conjugacy, it follows from the variational principle for entropy that image of the measure of maximal entropy under a conjugating map is again the measure of maximal entropy.

For part (3), observe that $\left\|D f_{(\lambda)}^{*}\right\| \leq \frac{1}{k}$ and thus $\left(D f_{(\lambda)}^{*}-I\right): C^{0}(K, \mathbb{R}) \rightarrow$ $C^{0}(K, \mathbb{R})$ is invertible. Moreover, the composition map $\left(f_{(\lambda)}, \pi\right) \mapsto f_{(\lambda)}^{*}(\pi)$ is $C^{1} \mathrm{cf}$. $\left[8\right.$, Lemma A.1]. Since the conjugacy $\pi_{(\lambda)}$ is defined implicitly by $f_{(\lambda)}^{*}\left(\pi_{(\lambda)}\right)-\pi_{(\lambda)}=$ 0 we can apply the Inverse Function Theorem to deduce that $(-\epsilon, \epsilon) \ni \lambda \mapsto \pi^{(\lambda)} \in$ $C^{0}(K, K)$ is $C^{1}$.

Remark. We easily see that $\pi_{(\lambda)}$ is actually $\alpha$-Hölder continuous with $\alpha=\frac{\log \beta}{\log d}$, where $\left|f_{(\lambda)}^{\prime}(\xi)\right| \geq \beta>1$, for all $t \in(-\epsilon, \epsilon)$.

This leads naturally to the following corollary to Proposition 1.1:

Corollary. For each $x \in K$ the map $(-\epsilon, \epsilon) \ni \lambda \mapsto \pi_{(\lambda)}(x) \in K$ is $C^{1}$.

Proof. The differentiability of $(-\epsilon, \epsilon) \ni \lambda \mapsto \pi_{(\lambda)} \in C^{0}(K, K)$ means that, by (1.1), for any linear functional $L: C^{0}(K, \mathbb{R}) \rightarrow \mathbb{R}$ we have that $(-\epsilon, \epsilon) \ni t \mapsto L\left(\pi_{(\lambda)}\right) \in \mathbb{R}$, is a $C^{1}$ map. We can choose that $L\left(\pi_{(\lambda)}\right)=\pi_{(\lambda)}(x)$ is the evaluation at the point $x$.

\section{Proof of Theorem 1}

The Haar measure $m_{(0)}$ is well known to be ergodic with respect to the linear map $f_{(0)}: K \rightarrow K$. In particular, we recall the following classical result.

Lemma 3.1 (Birkhoff Ergodic Theorem). There exists a set $X_{0} \subset K$ of full $m_{(0)}$ measure (i.e., Haar measure) such that for $\xi \in X_{0}$ and any continuous function $G: K \rightarrow \mathbb{R}$ we have that

$$
\lim _{N \rightarrow+\infty} \frac{1}{N} \sum_{i=0}^{N-1} G\left(f_{(0)}^{i} \xi\right)=\int G d m_{(0)} .
$$

Let $\lambda \in(-\epsilon, \epsilon)$ and $x \in K$. Given $F \in C^{0}(K, \mathbb{R})$, we can use $f_{(\lambda)}=\pi_{(\lambda)}^{-1} \circ f_{(0)} \circ \pi_{(\lambda)}$ to rewrite the Birkhoff averages

$$
\frac{1}{N} \sum_{i=0}^{N-1} F\left(f_{(\lambda)}^{i} x\right)=\frac{1}{N} \sum_{i=0}^{N-1}\left(F \circ \pi_{(\lambda)}^{-1}\right)\left(f_{(0)}^{i} \pi_{(\lambda)} x\right) \text { for } N \geq 1 .
$$


Moreover, if we write $\xi:=\pi_{(\lambda)}(x) \in X_{0}$ then applying the Lemma 3.1 with $G=$ $F \circ \pi_{(\lambda)}^{-1}$ we have that:

$$
\lim _{N \rightarrow+\infty} \frac{1}{N} \sum_{i=0}^{N-1}\left(F \circ \pi_{(\lambda)}^{-1}\right)\left(f_{(0)}^{i} \xi\right)=\int F \circ \pi_{(\lambda)}^{-1} d m_{(\lambda)}
$$

provided that $\pi_{(\lambda)}(x)$ is a typical point for the measure $m_{0}$, i.e., $\xi:=\pi_{(\lambda)}(x) \in X_{0}$.

Moreover, since $m_{(\lambda)}=\pi_{(\lambda)_{*}} m_{(0)}$ (by part (2) of Proposition 2.1) we have that

$$
\int F \circ \pi_{(\lambda)}^{-1} d m_{(\lambda)}=\int F d m_{(0)} .
$$

In particular, since $m_{(0)}$ is equal to Haar measure we see that for almost all $\lambda$ the image $\pi_{(\lambda)}(x)$ is in the set $X_{0}$ of full $m_{(0)}$ measure:

Lemma 3.2. Assume that $\left.\frac{\partial \pi_{(\lambda)}(x)}{\partial \lambda}\right|_{\lambda=0} \neq 0$. There there exists $0<\epsilon(x) \leq \epsilon$ such that for almost every $\lambda \in(-\epsilon(x), \epsilon(x))$ we have that $\pi_{(\lambda)}(x) \in X_{0}$.

Proof. For a given point $x$, we can choose $0<\epsilon(x) \leq \epsilon$ such that $\frac{\partial \pi_{(\lambda)}(x)}{\partial \lambda} \mid \neq 0$ for $t \in(-\epsilon(x), \epsilon(x))$. Then the map $(-\epsilon(x), \epsilon(x)) \ni t \mapsto \pi_{(\lambda)}(x)$ is a diffeomorphism onto its image. However, since $X_{0}$ has full $m_{(0)}$ (i.e., Haar) measure and the map $(-\epsilon(x), \epsilon(x)) \ni t \mapsto \pi_{(\lambda)}(x)$ has an absolutely continuous inverse, we deduce that

$$
\left\{\lambda \in(-\epsilon(x), \epsilon(x)): \pi_{(\lambda)}(x) \in X_{0}\right\}
$$

has full Lebesgue measure.

Finally, Theorem 1 follows by comparing Lemma 3.1 and Lemma 3.2.

Remark. We can choose sets of positive $m_{0}$ measure $X \subset K$ and $\Lambda_{x} \subset(-\eta, \eta)$, for $x \in X$ and $\eta>0$, of full Lebesgue measure $2 \eta$. such that the ergodic averages for $x \in X$ and $f_{(\lambda)}$, with $\lambda \in \Lambda_{x}$, correspond to the measure of maximal entropy $m_{(\lambda)}$. However, somewhat paradoxically, we cannot find $\lambda \neq 0$ for which $m_{(0)}\left\{x: \Lambda_{x} \ni\right.$ $\lambda\}>0$ since otherwise $m_{(\lambda)}$ would have to be equal to the absolutely continuous invariant measure for $f_{(\lambda)}$, which is patently not the case.

\section{Anosov diffeomorphisms}

Let $M$ be a $d$-dimensional compact manifold and let $f: M \rightarrow M$ be a transitive $C^{1}$ diffeomorphism. We call $f$ Anosov if there is a $C^{0}$ splitting $T M=E^{s} \oplus E^{u}$ such that there exist constants $C>0,0<\lambda<1$ such that $\left\|D f^{n} \mid E^{s}\right\| \leq C \lambda^{n}$ and $\left\|D f^{-n} \mid E^{n}\right\| \leq C \lambda^{n}$, for $n \geq 0$.

Example. Let $M=\mathbb{T}^{2}$ be a two dimensional torus and let $f_{(0)}: \mathbb{T}^{2} \rightarrow \mathbb{T}^{2}$ be a linear hyperbolic toral automorphism. To be more precise, we can consider

$$
f_{(\underline{0})}(x, y)=(2 x+y, x+y)(\bmod 1) .
$$

and a perturbation

$$
\begin{aligned}
f_{(\underline{\lambda})}(x, y) & =(2 x+y, x+y) \\
& +\left(\lambda_{1} \sin (2 \pi x)+\lambda_{2} \cos (2 \pi y), \lambda_{1} \cos (2 \pi x)+\lambda_{2} \sin (2 \pi y)\right)(\bmod 1),
\end{aligned}
$$

say, where $\underline{\lambda}=\left(\lambda_{1}, \lambda_{2}\right)$. We then have that $\operatorname{det}\left(D_{(\underline{0})} f_{(\underline{\lambda})}\right)=-\cos (2 \pi(x+y))$.

We require the following results on structural stability. Let $f_{(\lambda)}: M \rightarrow M$ be a $C^{1}$ family of transitive Anosov diffeomorphisms. Let $m_{(\underline{\lambda})}$ denote the unique measure of maximal entropy for $f_{(\underline{\underline{\lambda}})}$. Assume that for $f_{(\underline{0})}: M \rightarrow M$ the measure of maximal entropy is equivalent to the Riemannian volume on $M$. 


\section{Proposition 4.1 (Structural Stability for Anosov diffeomorphisms).}

(1) There exist conjugating homeomorphisms $\pi_{(\lambda)} \in C^{0}(M, M)$ such that

$$
\pi_{(\underline{\lambda})} f_{(\underline{\lambda})}=f_{(0)} \pi_{(\underline{\lambda})}, \text { for each } \underline{\lambda} \in(-\epsilon, \epsilon)^{d} \text {; }
$$

(2) $\pi_{(\underline{\lambda})_{*}} m_{(\underline{0})}=m_{(\underline{\lambda})}$ (i.e., for any continuous function $G: M \rightarrow \mathbb{R}$ we have that $\left.\int G \circ \pi_{(\underline{\lambda})} d m_{(\underline{0})}=\int G d m_{(\underline{\lambda})}\right)$

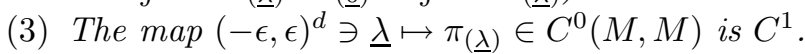

This can be deduced from the corresponding result for Anosov flows in [8] (cf. also [7] and [3]).

This leads naturally to the following:

Corollary. Let $x \in M$. Let $f_{(\underline{\lambda})}$ be a $C^{1}$ family of Anosov diffeomorphisms then the map $(-\epsilon, \epsilon)^{d} \ni \underline{\lambda} \mapsto \pi_{(\underline{\lambda})}(x) \in M$ is $C^{1}$.

This essentially appears as Part (b) of Theorem A.1 in [8]. Although the theorem is stated for Anosov flows and one dimensional perturbations, it is easily seen to apply in this case.

The natural analogues of Lemma 3.1 and Lemma 3.2 are:

Lemma 4.1. There exists a set $X_{0} \subset M$ of full $m_{(\underline{0})}$ measure (i.e., Haar measure) such that for $y \in X_{0}$ and any continuous $G: K \rightarrow \overline{\mathbb{R}}$ we have that

$$
\lim _{N \rightarrow+\infty} \frac{1}{N} \sum_{i=0}^{N-1} G\left(f_{(\underline{0})}^{i} y\right)=\int G d m_{(\underline{0})}
$$

Lemma 4.2. Assume that $\left.D \pi_{(\underline{\lambda})}(x)\right|_{\underline{\lambda}=\underline{0}}$ is non-singular. For almost every $\underline{\lambda} \in$ $(-\epsilon, \epsilon)^{d}$ we have that $\pi_{(\underline{\lambda})}(x) \in \bar{X}_{0}$.

Finally, we can compare Lemma 4.1 and Lemma 4.2 to deduce the following.

Theorem 2. Fix a point $x \in M$. For typical non-trivial families $f_{(\underline{\lambda})}: M \rightarrow M$ of Anosov diffeomorphisms we have that there exists $\epsilon=\epsilon(x)>0$ such that for almost all $\underline{\lambda} \in(-\epsilon, \epsilon)^{d}$, with respect to Lebesgue measure, we have that the $f_{(\underline{\lambda})}$-orbit of $x$ is normal, i.e., there exists a measure $m_{(\underline{\lambda})}$ on $M$ such that

$$
\lim _{n \rightarrow+\infty} \frac{1}{n} \sum_{i=1}^{n} g\left(f_{(\underline{\lambda})}^{i} x\right)=\int g(\xi) d m_{(\underline{\lambda})}(\xi) \quad \forall g \in C^{0}(K, \mathbb{R}) .
$$

In the above theorem, typically means the assumption that $\operatorname{det}\left(D_{\underline{0}} \pi_{(\underline{\lambda})}\right) \neq 0$.

Remark. From the proof of structural stability theorem [9], [8] can solve $D_{\underline{0}} \pi_{(\underline{\lambda})}=$ $\left(I-f_{*}\right)^{-1} D_{\underline{0}} f_{(\underline{\lambda})}$ where $f_{*} v(x)=D f v\left(f^{-1} x\right)$ and $\left(I-f_{*}\right)$ is invertible because of the hyperbolicity of $f$. In particular, providing the perturbation $\left.D f_{(\underline{\lambda})}(x)\right|_{\underline{\lambda}=\underline{0}}$ is non-singular we see that $\left.D \pi_{(\underline{\lambda})}(x)\right|_{\underline{\lambda}=\underline{0}}$ is non-singular. 


\section{Anosov flows}

There are completely analogous results for Anosov flows. Let $M$ be a $d$-dimensiona compact manifold and let $\phi_{t}: M \rightarrow M$ be a transitive $C^{1}$ flow. We call the flow Anosov if there is a $C^{0}$ splitting $T M=E^{0} \oplus E^{s} \oplus E^{u}$ such that

(i) $E^{0}$ is a one dimensional bundle tangent to the flow direction;

(ii) There exist constants $C>0, a>0$ such that $\left\|D \phi_{t} \mid E^{s}\right\| \leq C e^{-a t}$ and $\left\|D \phi_{t} \mid E^{s}\right\| \leq C e^{-a t}$, for $t \geq 0$.

Let $\phi_{(\underline{\lambda})}$ be a $C^{1}$ family of Anosov flows. Let $m_{(\underline{\lambda})}$ denote the measure of maximal entropy for $\phi_{(\underline{\lambda})}$. Assume that for $\phi_{(\underline{0})}$ the measure of maximal entropy is equivalent to the Riemannian volume on $M$.

We recall the following result.

\section{Proposition 5.1 (Structural Stability for Anosov flows).}

(1) There exist homeomorphisms $\pi_{(\underline{\lambda})} \in C^{0}(M, M)$ and changes of velocity $\rho_{(\underline{\lambda})}: M \rightarrow \mathbb{R}^{+}$. such that $\phi_{(\underline{\lambda})}$ is topologically conjugate by $\pi_{(\underline{\lambda})}$ to the reparameterization by $\rho_{(\underline{\lambda})}$ of $\phi_{(\underline{0})}$

(2) $\pi_{(\underline{\lambda})_{*}}\left(\rho m_{(\underline{0})}\right)=m_{(\underline{\lambda})}$ (i.e., for any continuous function $G: M \rightarrow \mathbb{R}$ we have that $\left.\int G \circ \pi_{(\underline{\lambda})} \rho d m_{(\underline{0})}=\int G d m_{(\underline{\lambda})}\right)$;

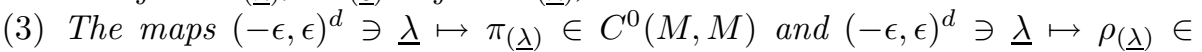
$C^{0}(M, \mathbb{R})$ are both $C^{1}$.

The above result was essentially proved in [8, Theorem A.1] (cf. also [7] and [3]) and, indeed, Proposition 3.1 in $\S 3$ was essentially already deduced from it. The only slight modification required is to deal with $d$-dimensional perturbations. In addition, part (2) on the conjugacy of flows is an easy consequence of well known results on the reparameterizations of flows.

A significant difference between the case of Anosov diffeomorphisms (Proposition 4.1) and Anosov flows (Proposition 5.1) is that in the latter case one cannot expect the conjugating map to be unique (due to the option of reparameterizing the flows). However, the images different conjugacies will lie on the same orbit.

Proposition 5.1 leads naturally to the following:

Corollary. Let $x \in M$. Let $\phi_{(\underline{\lambda})}$ be a $C^{1}$ family of Anosov flows then the map $(-\epsilon, \epsilon)^{d} \ni \underline{\lambda} \mapsto \pi_{(\underline{\lambda})}(x) \in M$ is $C^{\overline{1}}$.

This essentially appears as Part (b) of Theorem A.1 in [8]. Although the theorem is formally stated for Anosov flows and one dimensional perturbations, it is easily seen to apply in this case.

The natural analogues of Lemma 3.1 and Lemma 3.2 are:

Lemma 5.1. There exists a set $X_{0} \subset K$ of full $m_{(\underline{0})}$ measure such that for $y \in X_{0}$ and any continuous $G: K \rightarrow \mathbb{R}$ we have that

$$
\lim _{T \rightarrow+\infty} \frac{1}{T} \int_{0}^{T} G\left(\phi_{(\underline{0}) t} y\right) d t=\int G d m_{(\underline{0})} .
$$

Lemma 5.2. Assume that $\left.D \pi_{(\underline{\lambda})}(x)\right|_{\underline{\lambda}=0}$ is non-singular. For almost every $\underline{\lambda} \in$ $(-\epsilon, \epsilon)^{d}$ we have that $\pi_{(\underline{\lambda})}(x) \in \underline{X}_{0}$.

Finally, we can compare Lemma 5.1 and Lemma 5.2 to deduce the following. 
Theorem 3. Fix a point $x \in M$. For typical non-trivial families $\phi_{(\lambda)}$ we have that there exists $\epsilon=\epsilon(x)>0$ such that for almost all $\underline{\lambda} \in(-\epsilon, \epsilon)^{d}$, with respect to Lebesgue measure, we have that the $\phi_{(\underline{\lambda})}$ orbit of $x$ is normal, i.e., there exists a measure $m_{(\lambda)}$ on $M$ such that

$$
\lim _{T \rightarrow+\infty} \frac{1}{T} \int_{0}^{T} g\left(\phi_{(\underline{\lambda}), t} x\right)=\int g(\xi) d m_{(\underline{\lambda})}(\xi) \quad \forall g \in C^{0}(M, \mathbb{R}) .
$$

In the above theorem, typically means the assumption that $\operatorname{det}\left(D_{\underline{0}} \pi_{(\lambda)}\right) \neq 0$.

\section{RiEmAnN SURFACES}

We can consider an application of Theorem 3 on Anosov flows to geodesic flows of Riemann surfaces of higher genus. Let $V$ be a compact oriented surface with genus $g \geq 2$. The space of (Riemann) metrics $\rho$ of constant curvature $\kappa=-1$ form a space naturally identified with $\mathbb{R}^{6(g-1)}$. We can associate to any unit tangent vector $v \in T_{1} V$ a unique unit speed geodesic $\gamma_{v}: \mathbb{R} \rightarrow V$ which satisfies $\dot{\gamma}_{v}(0)=v$. It is natural to say that $v$ is normal if for any continuous function $F: T_{1} V \rightarrow \mathbb{R}$ we have that the $\operatorname{limit}_{T \rightarrow+\infty} \frac{1}{T} \int_{0}^{T} F\left(\dot{\gamma}_{v}(t)\right) d t$ exists.

The tangent space to the space of metrics at a fixed Riemann metric $\rho_{\underline{0}}$, say, can be identified with the space of real parts $\mathcal{R} Q$ of space of holomorphic quadratic differentials $Q$ (on the surface with metric $\rho_{0}$ ) [5].

Proposition 6.1. Let $V$ be a compact surface with a metric $\rho_{(\underline{0})}$ of constant curvature $\kappa=-1$. For any fixed $v \in T_{1} V$, we have that for almost all metrics in a sufficiently small neighbourhood $U$ of $\rho_{(\underline{0})}$ then $v$ is normal.

Proof. We can consider a parameterization $(-\epsilon, \epsilon)^{6(g-1)}$ of a neighbourhood of $\rho_{\underline{0}}$. We need to establish that the derivative of a conjugacy $\pi_{\underline{\lambda}}$ associated to the two geodesic flows satisfies $D_{\underline{0}} \pi_{(\underline{\lambda})}$ is non-singular. It is convenient to choose that if $v \in\left(T_{1} V\right)_{x}$ then $\pi_{(\underline{\lambda})}(v)$ lies in $\left\{\exp _{\rho_{0}} X: X \in(T V)_{x}, X \perp v,\|X\|<\epsilon\right\}$. Fathi and Flaminio [5], using the terminology of infinitessimal Morse Correspondences and the notation $\Xi:=D_{\underline{0}} \pi_{(\underline{\lambda})}$ showed that $\|\mathcal{R} Q\|^{2}=12\|\Xi\|^{2}[5$, Proposition 4.8]. From this result, the conclusion directly follows.

\section{REFERENCES}

1. K. Brucks and M. Misiurewicz, The trajectory of the turning point is dense for almost all tent maps., Ergodic Theory Dynam. Systems 16 (1996), 1173-1183.

2. H. Bruin, For almost every tent map, the turning point is typical., Fund. Math. 155 (1998), $215-235$.

3. G. Contreras,, Regularity of topological and metric entropy of hyperbolic flows., Math. Z. 210 (1992), 97-111.

4. B. Faller and C.-E. Pfister, A point is normal for almost all maps $\beta x+\alpha$ mod 1 or generalized $\beta$-maps., Preprint.

5. A. Fathi and L. Flaminio, Infinitesimal conjugacies and Weil-Petersson metric, Ann. Inst. Fourier (Grenoble) 43 (1993), 279-299.

6. A. Katok and B. Hasselblatt, Introduction to the modern theory of dynamical systems, Encyclopedia of Mathematics and its Applications, 54., Cambridge University Press,, Cambridge,, 1995..

7. A. Katok, G. Knieper, M. Pollicott, and H. Weiss,, Differentiability and analyticity of topological entropy for Anosov and geodesic flows., Invent. Math. 98 (1989), 581-597. 
8. R. de la Llave, J. Marco and R. Moriyon, Canonical perturbation theory of Anosov systems and regularity results for the Liv?ic cohomology equation, Ann. of Math. 123 (1986), 537-611.

9. J. Mather, Anosov Di?eomorphism, Appendix to Part I of "Di?erentiable Dynamical Systems" by S. Smale, Bull. Amer. Math. Soc., 73 (1967), 792-795. 